\title{
ETNOBOTÁNICA DE LA CULTURA TEOTIHUACANA
}

\author{
Mariana T. VázQuez-Alonso ${ }^{1,5}$, Robert Bye², Lauro López-Mata ${ }^{1}$, \\ Ma. Teresa P. Pulido-Salas ${ }^{3}$, Emily McClung de Tapia ${ }^{4}$ y Stephen D. $\mathrm{Koch}^{1}$ \\ 'Posgrado en Botánica, Colegio de Postgraduados, Montecillo, Estado de México, México \\ ${ }^{2}$ Instituto de Biología, Universidad Nacional Autónoma de México, México, D.F., México \\ ${ }^{3}$ Unidad de Recursos Naturales, Centro de Investigación Científica de Yucatán, Mérida, Yucatán, México \\ ${ }^{4}$ Instituto de Investigaciones Antropológicas, Universidad Nacional Autónoma de México, México, D.F., México \\ ${ }^{5}$ Autor para la correspondencia: vazquez.mariana@colpos.mx
}

\begin{abstract}
Resumen: El propósito de esta investigación fue obtener un listado de las plantas utilizadas por los antiguos habitantes de Teotihuacan prehispánico, a partir de la interpretación botánica y etnobotánica de registros arqueobotánicos e iconográficos. El registro resultante de plantas útiles para la cultura teotihuacana comprende 77 taxa y está basado en estudios publicados y reportes arqueológicos. Para conjuntar las evidencias de la presencia de las plantas con el contexto cultural se propuso un Índice de Importancia Cultural. Las evidencias directas son los restos arqueobotánicos identificados y el uso interpretado. Las evidencias indirectas son las representaciones en cerámica y mural, la necesidad de cultivar algunas especies y de importar otras. El maíz (Zea mays L.) es la especie con mayor importancia cultural. Los taxa reportados y especies relacionadas se buscaron en dos fuentes posteriores que describieran sus usos: el Códice Florentino y estudios etnobotánicos contemporáneos. Ambas fuentes mencionan más usos para los taxa listados. El uso medicinal es el menos identificado en Teotihuacan (ocho taxa); sin embargo, de acuerdo con los usos contemporáneos, el $57 \%$ de las especies pudo ser medicinal. La lista obtenida con interpretación etnobotánica de las posibles especies útiles para la cultura teotihuacana es de 125, incluyendo 17 especies interpretadas como cultivadas en Teotihuacan y 108 aprovechadas que pertenecen a diferentes tipos de vegetación. Veintiocho de las plantas fueron domesticadas en México. Este listado sirve como un acercamiento para entender la relación de la cultura teotihuacana con la flora local y regional.
\end{abstract}

Palabras clave: arqueobotánica, importancia cultural, Teotihuacan, zona arqueológica.

\begin{abstract}
The purpose of the research reported here was to develop a list of plants utilized by the ancient inhabitants of prehispanic Teotihuacan through the botanical and ethnobotanical interpretation of archaeobotanical and iconographic records. The resulting register of useful plants is comprised of 77 taxa, based on published studies and archaeological reports. A Cultural Significance Index was proposed in order to combine the information from different sources and evaluate their relative importance. Identified archaeobotanical remains and their interpretation based on archaeological excavations provide direct evidence for the use of plants. Representations in ceramics and mural paintings, along with evidence associated with cultivation or importation of some species, provide indirect evidence. Maize (Zea mays L.) is characterized as the most significant cultural species. The uses attributed to taxa reported from the archaeological record and related species were then consulted in the Florentine Codex, as well as contemporary ethnobotanical studies, both of which offer many additional references to the ways in which these plants were employed. Medicinal uses appear to be the least important at Teotihuacan (eight taxa); however, according to more recent sources, $57 \%$ of the species could have had medicinal applications. A total of 125 potentially useful plants were identified from Teotihuacan, based on the ethnobotanical interpretation, and includes 17 species definitely grown in Teotihuacan and 108 additional exploited taxa associated with different types of vegetation. Twenty-eight plants were domesticated in Mexico. This study contributes to an understanding of the relationship between the inhabitants of Teotihuacan to their local and regional flora.
\end{abstract}

Key words: archaeobotany, archaeological site, cultural significance, Teotihuacan.

$\mathbf{E}$ 1 desarrollo de las culturas prehispánicas en Mesoamérica estuvo asociado al cultivo de especies como calabaza, frijol y maíz. Mesoamérica es un área geográfica y cultural originalmente definida por Kirchhoff (1943) por la presencia de elementos como el empleo de bastón plantador (coa); la molienda del maíz cocido con ceniza o cal; el cultivo de cacao, chía y maguey; el uso ritual del hule; entre otros. También se ha propuesto como característica el sistema de cultivo de milpa (Zizumbo-Villareal et al., 2012). Esta área, dentro de la cual se desarrolló Teotihuacan, coincide con uno de los centros de domesticación y origen de plantas cultivadas (Sarukhán et al., 2009). La importancia de esta 
relación es que las plantas domesticadas por culturas nativas conforman la base del sistema alimentario mundial actual (Boege, 2010).

La cultura teotihuacana inició alrededor del año 100 a.C. en el valle de Teotihuacan, dentro de la cuenca de México, y fue la primera y más grande urbe precolombina. Alcanzó una máxima extensión territorial calculada en $22.5 \mathrm{~km}^{2}$ y una población de 125,000 habitantes (Millon, 1970). Es probable que su influencia en el exterior llegara hasta Honduras y Guatemala, según evidencia de cerámica y escultura. Empero, no después del año 650 , los principales templos fueron quemados y la ciudad quedó en ruinas (Cowgill, 2011). La población obtuvo sus recursos de la vegetación distribuida a lo largo de tres pisos altitudinales: zona montañosa con bosque de pino-encino y matorral de encino; zona de pie de monte y planicie aluvial con pastizal y matorral xerófilo; y zona de manantiales y orilla de lago con vegetación riparia y acuática (Castilla y Tejero, 1987; Hidalgo-Monroy, 1989; McClung, 2009). Las evidencias en las cuales se basa la reconstrucción de los tipos de vegetación propuestos son: la distribución contemporánea tanto de la vegetación como de los suelos, macro y micro restos botánicos recuperados de contextos arqueológicos y perfiles de suelo, y estudios geoarqueológicos y paleoambientales en áreas adyacentes a la cuenca de México (McClung et al., 2013).

Para haber sido una cultura tan importante, se desconoce información básica de ella; por ejemplo, no se sabe cómo se nombraban a sí mismos. El nombre Teotihuacan proviene de mitos relatados por culturas posteriores (Matos, 1995), escritos tanto en náhuatl como en español a la llegada de los españoles. Esto se debe a que cuando los mexicas llegaron a la cuenca de México, alrededor del año 1,300 (León-Portilla, 2005), la ciudad tenía más de 600 años de haber decaído.

Los teotihuacanos no dejaron registros escritos, por tanto, la reconstrucción de su forma de vida se ha hecho mediante la interpretación de los restos arqueológicos. Diversas exploraciones se hicieron en Teotihuacan desde la época prehispánica, entre sus visitantes posteriores se encuentran Carlos de Sigüenza y Góngora, la marquesa Calderón de la Barca, Desiré Charnay y Alexander von Humboldt. En 1907, el arqueólogo Leopoldo Batres inició la excavación de la Pirámide del Sol como parte de la reconstrucción de Teotihuacan (Bueno, 2010). Después, en 1917, Manuel Gamio realizó el proyecto "La Población del Valle de Teotihuacan", como parte de este estudio se elaboró una lista florística del Valle de Teotihuacan (Conzatti et al., 1979). En la década de 1960 René Millon dirigió el Teotihuacan Mapping Project y elaboró un mapa detallado de la ciudad en el periodo Clásico (Millon, 1970). Entre estos grandes proyectos y hasta la actualidad, se ha continuado con los trabajos arqueológicos. En cambio, los estudios arqueobotánicos no iniciaron al mismo tiempo que las excavaciones en Teotihuacan; la mayor parte se han realizado en los últimos 40 años. Durante este periodo han evolucionado las técnicas de análisis e identificación de restos vegetales, por lo que no se obtuvo información completa de las excavaciones iniciales. La interpretación de restos arqueobotánicos es la base del estudio de interacciones pasadas de poblaciones humanas y plantas (Schultes y von Reis, 1995; Cotton, 1996). Después de procesar los restos en laboratorio y compararlos con materiales en colecciones de referencia, arqueológicos y actuales, se proponen los posibles usos de una planta considerando el contexto en que fue encontrada (McClung, 1980). Por otro lado, las identificaciones taxonómicas en iconografía emplean resoluciones basadas en los caracteres biológicos de cada fitomorfo (Luna, 1996).

La importancia económica de una planta en arqueología se evalúa con base en su frecuencia relativa en diferentes muestras arqueológicas. Se puede considerar el número de restos por muestra, el número de muestras en que una especie es la común o la presencia en diferentes contextos. Además, se puede muestrear con un método experimental como el realizado en el valle de Tehuacán (Byers, 1967; McClung, 1980). No se han realizado estas evaluaciones de forma completa en Teotihuacan, debido a la gran variabilidad en el grado de conservación de los restos botánicos procedentes de diversos contextos excavados a través de las décadas.

Los estudios sobre el uso prehispánico de las plantas se enfocan usualmente en dos aspectos: el primero es el proceso de domesticación de las especies; el segundo es la descripción de los usos de las plantas encontradas en los manuscritos elaborados a la llegada de los españoles. A este respecto, existen cuatro fuentes importantes del siglo XVI que ilustran el conocimiento en este momento: (1) el Códice Florentino o Historia General de las cosas de la Nueva España, de Fray Bernardino de Sahagún, describe las propiedades de las plantas en su libro undécimo; (2) el Códice Cruz-Badiano o Libellus de medicinalibus indorum herbis, de Martín de la Cruz y Juan Badiano, documenta las hierbas medicinales de los indios; (3) la Historia Natural de la Nueva España, de Francisco Hernández, registra principalmente las plantas medicinales; y (4) las Relaciones Geográficas, son escritos solicitados por el rey de España, en ellos se describen las regiones y hacen referencia de algunas plantas utilizadas.

Los restos arqueobotánicos y las expresiones culturales (pintura mural y cerámica) no permiten una identificación a especie de todas las plantas empleadas por una cultura antigua. Debido a esto, el presente estudio buscó obtener un listado de las especies útiles para la cultura teotihuacana, por medio de una interpretación botánica y etnobotánica de los registros arqueobotánicos e iconográficos en estudios realizados por especialistas, sobre materiales provenientes de la Zona Arqueológica de Teotihuacan.

\section{Material y métodos}

Revisión de las plantas utilizadas por los teotihuacanos. Para compilar el uso de la flora por la cultura teotihuacana se 
revisaron publicaciones, además de informes arqueológicos reportados al Instituto Nacional de Antropología e Historia (INAH) en torno a Teotihuacan. Se encontraron identificaciones de plantas útiles en sólo 45 investigaciones realizadas entre los años 1977 al 2012. Las citas de estos trabajos se encuentran en el apartado de arqueología del Cuadro 1. En las investigaciones, los taxa fueron identificados a partir de: (1) restos arqueobotánicos procedentes de excavaciones, como son los restos macrobotánicos (semillas, partes de frutos, madera carbonizada, tallos) y los restos microbotánicos (polen y fitolitos); y (2) la interpretación de representaciones de fitomorfos en murales y cerámica.

Los usos de las plantas fueron determinados por especialistas, al interpretar el contexto en que encontraron los restos. Los nombres científicos de los taxa son los mismos citados por los autores. Para clasificar los usos de las plantas, se tomó en cuenta el estándar de datos de colecciones de botánica económica (Cook, 1995). Las categorías empleadas son: comestible (Co), combustible (Cb), material (Ma), medicinal (Me) y uso social (Soc).

Índice de importancia cultural. La importancia o valor relativo de las especies o familias de plantas para la sociedad se ha determinado mediante el empleo de índices etnobotánicos con un enfoque cultural (Hoffman y Gallaher, 2007). Para capturar diferentes aspectos de la importancia de una especie, es posible combinar índices que midan tanto valores culturales, así como prácticos y económicos (ReyesGarcía et al., 2006). La mayoría de estos índices se basan en reportes de usos por informantes locales, método que no es aplicable en este estudio ya que se buscan usos antiguos.

El Índice de Importancia Cultural (IIC) propuesto en este trabajo se basó en dos índices: el índice de valor de importancia etnobotánica de Lajones y Lema (1999), y el índice de importancia cultural de Suárez et al. (2012). El IIC se calculó para cada taxón en el listado de plantas útiles teotihuacanas, mediante la expresión:

$$
\mathrm{IIC}=(\mathrm{U}+\mathrm{PRB}+\mathrm{R}+\mathrm{C}+\mathrm{I}) / 5
$$

donde, IIC es el Índice de Importancia Cultural. Las variables son: usos $(\mathrm{U})$, presencia de restos botánicos (PRB), representaciones (R), cultivada (C) e importada (I). El término de importación se emplea para nombrar a una especie que no es nativa de la región y que pudo introducirse por diferentes razones, sea o no mediante el comercio. Las variables están calculadas por medio de un número expresado en términos relativos respecto a sí mismos y multiplicado por el número de categorías de cada variable. $\mathrm{U}=\mathrm{u} \times 5$, u es el número de usos de la planta (1 a 5), multiplicado por cinco; $\mathrm{PRB}=$ prb $\times 3$, prb es la presencia de restos botánicos en 16 excavaciones arqueológicas, los valores están dados en tres categorías: baja (1), media (2), alta (3), multiplicado por tres; $\mathrm{R}=\mathrm{r} \times 2$, $\mathrm{r}$ es la representación en cerámica (1), en murales (1) o en ambas (2), multiplicado por dos; $\mathrm{C}=\mathrm{c} \times 1$, c es si fue cultivada (1), multiplicado por uno; e I = i $\times 1$, i es si fue importada (1), multiplicado por uno. El IIC es una suma de los componentes y después dividida entre cinco, que es el número de variables que lo componen (U, PRB, R, C e I).

Comparación con fuentes históricas y estudios recientes. Poco después de la conquista, fueron descritos los usos prehispánicos de las plantas de las culturas presentes en ese momento. Por ser una cultura anterior, para Teotihuacan no se tienen fuentes escritas. Con el objetivo de reconocer posibles usos que no pudieron ser interpretados a partir de los registros arqueológicos, se hizo una revisión de los usos reportados en fuentes posteriores para los mismos taxa identificados con un uso en Teotihuacan. Para esto, se buscaron los usos de las plantas en el Códice Florentino (Dibble y Anderson, 1963; Sahagún, 1980; Estrada, 1989) y en estudios etnobotánicos contemporáneos de los estados de la cuenca de México (Conzatti et al., 1979; Caballero y Cortés, 19822013). El número de especies por categoría de uso para cada fuente se presenta en una tabla comparativa. El análisis de frecuencias se elaboró con el procedimiento PROC FREQ de SAS (SAS Institute Inc., 2010).

Propuesta de posibles especies útiles para la cultura teotihuacana. Para obtener un listado de especies teotihuacanas potencialmente útiles, a partir de los registros identificados por restos arqueobotánicos e iconografía, se hizo una interpretación botánica. Las referencias en los estudios sobre plantas útiles teotihuacanas se encuentran en diferentes niveles taxonómicos; esto significa que no todos los taxa están identificados a nivel de especie. Lo anterior debido a que muchos de los restos botánicos se encuentran carbonizados y fragmentados. Además, se actualizó la nomenclatura taxonómica para organizar todos los taxa a una nomenclatura contemporánea.

La propuesta de especies a las que pueden pertenecer esos taxa se basó en 13 estudios botánicos y 21 etnobotánicos (apartados de botánica y etnobotánica en Cuadro 1). La principal obra de referencia para plantas actuales es la Flora fanerogámica del valle de México (Calderón de Rzedowski y Rzedowski, 2005). El sistema de clasificación de especies de plantas con flores utilizado en el listado fue el propuesto por Takhtajan (1997). Los nombres de las autoridades se verificaron en el Catálogo de autores de plantas vasculares de México (Villaseñor et al., 2008).

\section{Resultados}

Revisión de las plantas utilizadas por los teotihuacanos. Los restos arqueobotánicos y las representaciones en murales y cerámica muestran que además de usar plantas para su alimentación, los teotihuacanos requerían de especies para leña, construcción, fabricación de utensilios, vestido, obtención de mucílagos, resinas y látex, plantas con fines alucinógenos y 
Cuadro 1. Fuentes bibliográficas revisadas para completar el listado de especies teotihuacanas y su posterior interpretación botánica y etnobotánica. Las citas completas están disponibles solicitándolas a la autora principal.

\begin{tabular}{cl}
\hline Tema & \multicolumn{1}{c}{ Autores } \\
\hline Arqueología & Adriano-Morán y McClung, 2008; Angulo, 1996; Cabrera et al., 1991; Cabrera, 2006; Carreón, 2004; Cásales, \\
& 1994; Cowgill, 1997; Doménech-Carbó, 2012; de la Fuente, 2006; González, 1986; González et al., 1993; \\
& Gónzalez y Sánchez, 1991; Heyden, 1983; Hidalgo-Monroy, 1989; Langley, 2008; Lot y Corona, 1977; Luna, \\
& 2006; Manzanilla, 1993a, 1993b, 1995, 2003, 2008, 2011, 2012; Manzanilla et al., 2009; Manzanilla y Arrellín, \\
& 1999; McClung, 1977, 1979, 1987, 1989, 1990, 2003, 2009; McClung y Tapia, 1993; McClung y Rodríguez, \\
& 1995; Montúfar, 1994; Nichols et al., 1991; Pérez, 2011; Rattray, 1997; Rivas, 2001; Sugiura, 2008; Sugiyama, \\
& 2001; Uriarte, 1996, 2006; Vela, 2011. \\
\hline Botánica & Calderón de Rzedowski y Rzedowski, 2005; Castilla y Tejero, 1987; Clemants y Mosyakin, 2003a; Clemants y \\
& Mosyakin, 2003b; Conzatti et al., 1979; Farjón A. et al., 1997; Fryxell, 1978, 1993; Gómez, 2008; Martínez, \\
& 1998; Reyes-Agüero et al., 2005; Standley, 1920-1926; Wilson y Heiser, 1979. \\
\hline Etnobotánica & Cahill, 2003; Campos et al., 2008; Galwey, 1995; García, 2007; Hernández-Bermejo y León, 1992; Kato et al.,, \\
& 2009; Kennedy, 1995; León, 2000; Linares y Bye, 2008; Mapes, 1990; Mera et al., 2011; Nuez et al., 2000; \\
& Pennington y Sarukhán, 2005; Purata, 2008; Sánchez, 2008; Santiaguillo y Blas, 2009; Sauer, 1995; Scheinvar \\
& et al., 2009; Téliz y Mora, 2007; Vázquez-Yanes et al., 1999; Wendel, 1995. \\
\hline
\end{tabular}

medicinales, otras como parte de ofrendas y entierros; algunas de ellas eran importadas de regiones más cálidas.

El listado de plantas útiles teotihuacanas comprende 77 taxa (según la nomenclatura reportada por los autores), pertenecientes a 54 géneros y 34 familias (Apéndice 1). De las cinco categorías de uso, la comestible presenta la mayor riqueza e incluye 33 taxa, seguido de usos sociales con 24 , combustible con 20 , materiales con 16 y medicinal con ocho. El 52\% de las especies es identificado con más de un uso. Los taxa con más usos fueron Agave, Chenopodium, Opuntia, Pinus, Prunus y Zea mays.

Índice de Importancia Cultural. La cantidad de restos botánicos no precisamente demuestra mayor o menor uso, puede estar relacionada a la densidad y perdurabilidad de las partes. Debido a esto, el IIC propuesto y el análisis de la información que contiene intentan proporcionar una aproximación acerca de las plantas útiles para la cultura teotihuacana.

Cuadro 2. Valores de los componentes del Índice de Importancia Cultural para los taxa reportados en Teotihuacan $(U=$ uso, $P R B=$ presencia de restos botánicos, $\mathrm{R}$ = representaciones, $\mathrm{C}=$ cultivada, I = importada, IIC = índice de importancia cultural).

\begin{tabular}{|c|c|c|c|c|c|c|}
\hline Taxa & $\mathbf{U}$ & PRB & $\mathbf{R}$ & C & I & IIC \\
\hline Zea mays & 16.0 & 12.2 & 12.9 & 6.7 & 11.1 & 11.8 \\
\hline Opuntia spp. & 16.0 & 12.2 & 6.5 & 6.7 & 0.0 & 8.2 \\
\hline Agave spp. & 21.3 & 4.1 & 6.5 & 0.0 & 0.0 & 6.4 \\
\hline Chenopodium sp. & 16.0 & 8.1 & 0.0 & 6.7 & 0.0 & 6.1 \\
\hline Prunus serotina subsp. capuli & 21.3 & 8.1 & 0.0 & 0.0 & 0.0 & 5.9 \\
\hline Theobroma сасао & 5.3 & 0.0 & 12.9 & 0.0 & 11.1 & 5.9 \\
\hline Quercus spp. & 10.6 & 12.2 & 6.5 & 0.0 & 0.0 & 5.9 \\
\hline Cucurbita spp. & 5.3 & 4.1 & 12.9 & 6.7 & 0.0 & 5.8 \\
\hline Pinus spp. & 16.0 & 12.2 & 0.0 & 0.0 & 0.0 & 5.6 \\
\hline (copal) & 5.3 & 4.1 & 6.5 & 0.0 & 11.1 & 5.4 \\
\hline Gossypium sp. & 5.3 & 4.1 & 6.5 & 0.0 & 11.1 & 5.4 \\
\hline Persea sp. & 10.6 & 4.1 & 0.0 & 0.0 & 11.1 & 5.2 \\
\hline Amaranthus spp. & 10.6 & 8.1 & 0.0 & 6.7 & 0.0 & 5.1 \\
\hline Capsicum sp. & 10.6 & 8.1 & 0.0 & 6.7 & 0.0 & 5.1 \\
\hline Physalis sp. & 10.6 & 8.1 & 0.0 & 6.7 & 0.0 & 5.1 \\
\hline Amaranthus leucocarpus & 5.3 & 0.0 & 12.9 & 6.7 & 0.0 & 5.0 \\
\hline Castilla elastica & 5.3 & 0.0 & 6.5 & 0.0 & 11.1 & 4.6 \\
\hline Phaseolus vulgaris & 5.3 & 4.1 & 6.5 & 6.7 & 0.0 & 4.5 \\
\hline Ficus sp. & 5.3 & 4.1 & 0.0 & 0.0 & 11.1 & 4.1 \\
\hline Lagenaria siceraria & 5.3 & 4.1 & 0.0 & 0.0 & 11.1 & 4.1 \\
\hline Restantes 58 taxa & 292.6 & 178.4 & 103.2 & 40.0 & 11.1 & 125.1 \\
\hline Total & 500.0 & 300.0 & 200.0 & 100.0 & 100.0 & 240.0 \\
\hline
\end{tabular}


Los trece primeros lugares en importancia cultural (Cuadro 2) concentran el $34 \%$ de este índice. Nueve de ellos son comestibles (cinco son cultivados), cuatro son árboles aprovechados de vegetación natural y cinco se importan de otras regiones. El maíz (Zea mays) es la especie con un mayor IIC; tiene tres usos, está presente en la mitad de los sitios, se encuentra representada en cerámica y pintura mural, fue cultivada y, además, es una especie que fue importada.

Comparación con fuentes históricas y estudios recientes. Los taxa reportados como útiles para la cultura teotihuacana y especies relacionadas se buscaron en fuentes posteriores que describieran los usos de plantas para plantear hipótesis respecto a los usos potenciales durante periodos más antiguos (Cuadro 3). Se tomaron dos momentos: el primero, representado por el Códice Florentino, el cual recopila usos prehispánicos años después de la conquista en el siglo XVI; y el segundo se tomó de estudios etnobotánicos contemporáneos desde 1900 a la fecha.

Los 121 taxa tienen 99 usos en Teotihuacan, 112 en el Códice Florentino y 200 en los estudios recientes. Los usos, en las tres fuentes, con más especies son: comestible y me-

Cuadro 3. Usos de las plantas teotihuacanas reportados en el Códice Florentino y en estudios etnobotánicos contemporáneos. Usos: $\mathrm{Cb}=$ combustible, $\mathrm{Co}=$ comestible, $\mathrm{Ma}=$ material, $\mathrm{Me}=$ medicinal, $\mathrm{Soc}=$ social. Datos: $\mathrm{A}$ = frecuencia (número de especies con ese uso), $\mathrm{B}=$ porcentaje con relación al total de usos (413 usos), C = porcentaje por fila (categoría de uso), D = porcentaje por columna (fuente), $\mathrm{E}=$ total de frecuencia, $\mathrm{F}=$ total de porcentaje.

\begin{tabular}{|c|c|c|c|c|c|}
\hline \multirow[b]{2}{*}{ Usos } & \multirow[b]{2}{*}{ Datos } & \multicolumn{3}{|c|}{ Fuentes } & \multirow[b]{2}{*}{ Tota } \\
\hline & & Teotihuacan & $\begin{array}{r}\text { Códice } \\
\text { Florentino }\end{array}$ & $\begin{array}{l}\text { Estudios con- } \\
\text { temporáneos }\end{array}$ & \\
\hline \multirow[t]{4}{*}{$\mathrm{Cb}$} & A & 20 & 7 & 18 & 45 \\
\hline & B & 4.8 & 1.7 & 4.4 & 10.9 \\
\hline & $\mathrm{C}$ & 44.4 & 15.6 & 40.0 & \\
\hline & $\mathrm{D}$ & 19.8 & 6.3 & 9.0 & \\
\hline \multirow[t]{4}{*}{ Co } & A & 33 & 34 & 45 & 112 \\
\hline & B & 8.0 & 8.2 & 10.9 & 27.1 \\
\hline & $\mathrm{C}$ & 29.5 & 30.4 & 40.2 & \\
\hline & $\mathrm{D}$ & 32.7 & 30.4 & 22.5 & \\
\hline \multirow[t]{4}{*}{ Ma } & A & 16 & 13 & 36 & 65 \\
\hline & B & 3.9 & 3.1 & 8.7 & 15.7 \\
\hline & $\mathrm{C}$ & 24.6 & 20.0 & 55.4 & \\
\hline & $\mathrm{D}$ & 15.8 & 11.6 & 18.0 & \\
\hline \multirow[t]{4}{*}{ Me } & A & 8 & 32 & 72 & 112 \\
\hline & B & 1.9 & 7.7 & 17.4 & 27.1 \\
\hline & $\mathrm{C}$ & 7.1 & 28.6 & 64.3 & \\
\hline & $\mathrm{D}$ & 7.9 & 28.6 & 36.0 & \\
\hline \multirow[t]{4}{*}{ Soc } & A & 24 & 26 & 29 & 79 \\
\hline & B & 5.8 & 6.3 & 7.0 & 19.1 \\
\hline & $\mathrm{C}$ & 30.4 & 32.9 & 36.7 & \\
\hline & $\mathrm{D}$ & 23.8 & 23.2 & 14.5 & \\
\hline \multirow[t]{2}{*}{ Total } & E & 101 & 112 & 200 & 413 \\
\hline & $\mathrm{F}$ & 24.1 & 27.3 & 48.7 & 100 \\
\hline
\end{tabular}

dicinal (27\%), social (19\%), material (16\%) y por último, combustible (11\%). El uso combustible tiene un mayor número de taxa reportados para Teotihuacan que para las otras fuentes. La proporción en los usos cambia entre Teotihuacan y el Códice Florentino; el comestible es el mayor para ambos, pero el medicinal es mayor en el Códice Florentino. En los estudios etnobotánicos contemporáneos, el principal uso es medicinal.

Propuesta de las especies útiles para la cultura teotihuacana. Los taxa reportados en arqueología como plantas útiles corresponden, algunos a especies identificadas, otros a géneros y algunos otros a especies no identificadas de alguna familia. Las especies que pueden corresponder con estos taxa son 125 , las cuales son el número probable de especies útiles teotihuacanas conocidas hasta el momento (Apéndice 1).

Las especies corresponden a 37 familias, de las cuales, Agavaceae, Cactaceae, Pinaceae y Solanaceae son las mejor representadas. Los géneros más frecuentes son: Agave, Opuntia, Pinus y Quercus. Tomando de referencia los tipos de vegetación reconstruidos propuestos por McClung et al. (2013), el tipo de vegetación al que pertenecen más especies es el matorral xerófilo (38\%), seguido por el bosque de pino-encino (22\%).

La distribución de especies dentro de las categorías comestible, combustible, material y social es similar, con un rango entre 21 y $24 \%$. La categoría con el menor número de especies es la medicinal. Del total de especies, sólo el 33\% tiene más de un uso.

Tres de las especies se encuentran en la Norma Oficial Mexicana NOM-059 (SEMARNAT, 2010): Cupressus lusitanica Mill. (sujeta a protección especial), Mammillaria wiesingeri Boed. subsp. wiesingeri (endémica, amenazada) y Nymphaea mexicana Zucc. (amenazada). Lo que indica que la distribución y abundancia de las especies dentro del valle de Teotihuacan pudo haberse modificado y que en la actualidad no se vea el mismo paisaje que en la era antigua.

\section{Discusión}

La base de este trabajo son los restos arqueobotánicos y la iconografía de plantas interpretados con algún uso antiguo. El utilizar estos datos para reconstruir la etnobotánica de la cultura teotihuacana tiene limitantes, como la preservación de los materiales para su identificación y la dificultad de la interpretación de su uso. Además de estas cuestiones propias de la disciplina, existe un desfase en el acceso a los datos. En algunos casos los taxa identificados quedan desvinculados de la interpretación general de la excavación. En otros casos las identificaciones forman parte sólo de reportes técnicos que no son publicados (ver Manzanilla, 2011). Por tanto, muchos de los datos de especies útiles pierden relevancia y quedan dispersos. 
Los taxa reportados como útiles dentro de la cultura teotihuacana pertenecen a cinco categorías de uso, siendo el principal uso el comestible, después el social, combustible, material y por último, el medicinal. Esta no es la proporción usual de plantas útiles reportadas en estudios etnobotánicos; las plantas medicinales suelen ser las más numerosas (Navarro y Avendaño, 2002; Monroy-Ortiz y Monroy, 2004). En cambio, en Teotihuacan pocas especies se han podido interpretar con este último uso, posiblemente debido a que los procesos de preparación y consumo de plantas para usos medicinales disminuye la posibilidad de que se conserven restos. El empleo de nuevas técnicas, como el análisis de fitolitos, gránulos de almidón o alcaloides, apenas se inician en Teotihuacan y podrían ayudar a relacionar algunas especies con implementos de preparación de plantas, tanto comestibles como medicinales. El uso ornamental, también reportado como importante en comunidades, no está registrado para Teotihuacan por la dificultad de la interpretación de este uso en los restos arqueobotánicos. Aun siendo ocupadas las formas vegetales de forma decorativa, en la mayoría de los murales no se ha interpretado el uso ornamental de una especie en particular.

En estudios arqueobotánicos en Teotihuacan, se ha atribuido una importancia económica a un taxa cuando ocurre en muestras que representan toda la secuencia cronológica, procede de varios sectores residenciales de la ciudad y de diferentes contextos arqueológicos (McClung, 1980). De esta forma, se distinguen las especies con una buena distribución en las muestras; sin embargo, géneros con una conocida importancia posterior como Cucurbita y Phaseolus, están escasamente presentes como restos arqueológicos.

Con el Índice de Importancia Cultural, propuesto en este trabajo, se busca reunir toda la información disponible de las plantas útiles en Teotihuacan, al incluir evidencias físicas: el número de sitios de excavación en que está presente un resto botánico y el número de usos que fueron interpretados para el taxa. Además de evidencias culturales: el manejo agrícola de cultivo, el esfuerzo de importación y la representación en iconografía mural o cerámica. La ubicación de los taxa en todos los ámbitos nos da una idea de su presencia en la ciudad, que aun así no es una estimación de su uso real. El IIC expresa la importancia de una especie en función de un conjunto de atributos; de esta forma, especies notorias por su uso ritual como el copal, aparecen junto a especies de uso cotidiano. Por ejemplo, los pinos fueron utilizados para leña y madera (construcción), los restos de madera carbonizada demuestran su presencia en casi todos los sitios, lo que muestra un uso difundido; a diferencia del copal, que se encuentra como resina en incensarios y es interpretado en murales como un material portado por sacerdotes. El índice reúne la evidencia sobre plantas, encontrada hasta el momento, en estudios de la zona arqueológica sin hacer una valoración por el conocimiento posterior de las plantas útiles. En el índice, a todos los usos se les asignó el mismo valor, a diferencia de otros índices donde dan mayor valor al alimenticio que al ritual (Lajones y Lema, 1999). Por el tipo de sociedad que se estudió, con un fuerte vínculo con la naturaleza, en donde incluso asocian plantas con dioses, como el amamalácotl con Tláloc, no se puede demeritar el valor de una planta ritual.

La pintura mural cubría toda la ciudad desde una forma compleja a una más simple. Una pequeña parte se conservó y ahora es fuente de entendimiento de la cultura. Es difícil discernir si están representando su realidad, la relación que tienen con el ambiente o su cosmogonía. Las plantas están representadas a veces en su forma biológica y otras veces de una forma simbólica. Aun así, se puede interpretar un uso para el fitomorfo, pero la mayor importancia es la representación de una especie particular; ya que por el contrario, el icono de la flor de cuatro pétalos es una forma genérica de flor que tiene una carga simbólica (Heyden, 1983; Angulo, 1996). En el caso del maíz, aparece de forma repetida en el mural Tlalocan de Tepantitla, tal vez representando un cultivo; en cambio, las mazorcas de cuatro colores portadas por el Tlaloc de Zacuala están mostrando su cosmogonía, dándole una importancia ritual.

Por las características de cada fuente histórica, la información es diferente. Los reportes de usos son mayores en los estudios recientes, mostrando los usos que no pueden verse en los registros arqueológicos para Teotihuacan. El uso donde es más clara la diferencia es el medicinal, que es difícil interpretar en restos arqueobotánicos, pero citado en el Códice Florentino y en estudios recientes para las mismas especies con otro uso en Teotihuacan. De manera opuesta, los reportes de especies útiles como combustible son un poco mayor para Teotihuacan, debido a que la madera carbonizada puede analizarse incluso a nivel de especie.

Al comparar cada especie en las fuentes se notan diferencias en la parte de la planta utilizada. Entre los restos arqueobotánicos que mejor se preservan, se tienen las semillas y la madera, estos refieren diferentes tipos de usos. Por ejemplo, las semillas pueden mostrar consumo y la madera carbonizada en fogones el uso de las plantas como combustible. De esta forma se pierde la evidencia del uso de una planta en verde o del uso medicinal de un árbol, los cuales son mencionados en las descripciones escritas posteriores.

Durante siglos, las comunidades en todo el mundo han aprendido, usado y transferido los conocimientos tradicionales sobre la biodiversidad local y la forma en que puede ser utilizada para una variedad de propósitos importantes (CDB, 2011). En México, diversas etnias del país han conservado y enriquecido el conocimiento tradicional, muestra del legado cultural que dejaron las culturas prehispánicas (GómezPompa, 1993). Este conocimiento se ha recabado y evaluado mediante estudios etnobotánicos en las comunidades.

La intención de esta investigación fue obtener un listado de plantas útiles de una cultura antigua que perduró por aproximadamente 700 años y decayó hace más de 1,000 
años. Cuando se habla de culturas prehispánicas se suelen nombrar las especies domesticadas. La lista de posibles especies útiles para la cultura teotihuacana incluye 28 plantas domesticadas en México, de acuerdo con Hernández-X. (1998), de ellas sólo 17 se han interpretado como cultivadas en Teotihuacan. Además, se aprovecharon 116 especies provenientes de diferentes tipos de vegetación.

Este listado sirve como un acercamiento para entender la relación de la cultura teotihuacana con la flora, local y regional. Desde una perspectiva académica, la nomenclatura botánica actualizada proporciona un marco taxonómico para la comparación de las etnofloras actualmente documentadas. El listado, junto con el índice de importancia cultural, permitirán una valorización rápida de los futuros descubrimientos arqueobotánicos de uno de los sitios arqueológicos americanos más importantes. Los resultados de nuestro análisis (como el de la baja representación por categoría etnocéntrica de plantas medicinales), enfatizan la necesidad de aplicar nuevos muestreos y técnicas diagnósticas para probar las hipótesis de la utilización diferencial de plantas. La comparación de las etnofloras entre diferentes sitios, especialmente entre centros ceremoniales como Teotihuacan y sitios agrícolas, puede revelar nuevas perspectivas sobre el papel de los recursos vegetales locales, así como de plantas y productos botánicos intercambiados. Desde la perspectiva de la difusión y el manejo de los recursos culturales, la lista botánica proporciona un marco de referencia para la interpretación antropológica contemporánea de evidencia arqueológica, para que los visitantes de la zona arqueológica (tanto nacionales como extranjeros), e investigadores puedan entender los cambios de las relaciones entre las sociedades y la naturaleza, en Mesoamérica, a lo largo de dos mil años.

\section{Agradecimientos}

El primer autor agradece al Colegio de Postgraduados, al Consejo Nacional de Ciencia y Tecnología por el financiamiento otorgado para estudios de maestría y al Consejo Mexiquense de Ciencia y Tecnología por la beca para término de tesis. También al personal de la Zona Arqueológica de Teotihuacan por su interés en un estudio etnobotánico. Los autores agradecen las revisiones críticas de dos árbitros anónimos cuyos comentarios y sugerencias mejoraron sustancialmente el artículo.

\section{Literatura citada}

Angulo V.J. 1996. Teotihuacán. Aspectos de la cultura a través de su expresión pictórica. En: de la Fuente B. Coord. La Pintura Mural Prehispánica en México, I Teotihuacan, Tomo II, pp. 65186. Instituto de Investigaciones Estéticas, Universidad Nacional Autónoma de México, México, D.F.

Boege S.E. 2010. El Patrimonio Biocultural de los Pueblos Indígenas de México. Hacia la Conservación In Situ de la Biodiversidad y Agrobiodiversidad en los Territorios Indígenas. Instituto
Nacional de Antropología e Historia, Comisión Nacional para el Desarrollo de los Pueblos Indígenas, México, D.F.

Bueno C. 2010. Teotihuacan: showcase for the Centennial. En: Berger D. y Grant W.A. Eds. Holiday in Mexico: Critical Reflections on Tourism and Tourist Encounters, pp. 54-76, Duke University Press, Durham.

Byers D.S. 1967. The Prehistory of the Tehuacan Valley. Vol. 1: Environment and Subsistence. Robert S. Peabody Foundation, University of Texas Press, Austin.

Caballero J. y Cortés Z.L. 1982-2013. Base de Datos Etnobotánicos de Plantas de México (BADEPLAM). Instituto de Biología, Universidad Nacional Autónoma de México, México, D.F.

Calderón de Rzedowski G. y Rzedowski J. 2005. Flora Fanerogámica del Valle de México. $2^{\mathrm{a}}$ ed. Instituto de Ecología A.C., Comisión Nacional para el Conocimiento y Uso de la Biodiversidad, Pátzcuaro.

Castilla H.M. y Tejero D.J.D. 1987. Flora y vegetación del Cerro Gordo (San Juan Teotihuacan) y regiones aledañas, Valle de México, México. Biotica 12:231-255.

CDB. Convenio sobre la Diversidad Biológica. 2011. Conocimiento tradicional. Convenio sobre la Diversidad Biológica: ABS. Secretaría del Convenio sobre la Diversidad Biológica, Programa de las Naciones Unidas para el Medio Ambiente, Montreal.

Conzatti C., Herrera M., Ochoterena I. y Noriega J.M.. 1979. La flora. En: Gamio M. Ed. La Población del Valle de Teotihuacan, pp. 24-42, Primera parte, ambiente físico-biológico, Edición facsimilar, Instituto Nacional Indigenista, México, D.F.

Cook F.E.M. 1995. Economic Botany Data Collection Standard. International Working Group on Taxonomic Databases for Plant Sciences. Royal Botanic Gardens, Kew, Londres.

Cotton C.M. 1996. Ethnobotany: Principles and Applications. John Wiley \& Sons, Chichester.

Cowgill G.L. 2011. A speculative history of Teotihuacan. $5^{a}$ Mesa Redonda de Teotihuacan. Teotihuacan: Investigaciones Recientes Centro y Periferia. Ponencia magistral. Instituto Nacional de Antropología e Historia, Consejo Nacional para la Cultura y las Artes, Centro de Estudios Teotihuacanos. Teotihuacán.

Dibble C.E. y Anderson A.J.O. 1963. Florentine Codex. General History of the Things of New Spain, Fray Bernardino de Sahagún. Book II Earthly Things. Vol. XII. The School of American Research, The University of Utah Press, Santa Fe.

Estrada L.E.I.J. 1989. El Códice Florentino, su Información Etnobotánica. Colegio de Postgraduados, Chapingo.

Gómez-Pompa A. 1993. Las raíces de la etnobotánica mexicana. Acta Biologica Panamensis 1:87-100.

Hernández-X. E. 1998. Aspectos de la domesticación de plantas en México: una apreciación personal. En: Ramamoorthy T.P., Bye R., Lot A. y Fa. J. Eds. Diversidad Biológica de México: Orígenes y Distribución, pp. 715-735, Instituto de Biología, Universidad Nacional Autónoma de México, México, D.F.

Heyden D. 1983. Mitología y Simbolismos de la Flora en el México Prehispánico. Universidad Nacional Autónoma de México, Instituto de Investigaciones Antropológicas, México, D.F.

Hidalgo-Monroy N. 1989. Uso de la flora y la fauna en una unidad habitacional de Teotihuacan, Estado de México. Tesis licenciatura, Escuela Nacional de Antropología e Historia, México, D.F. 85 pp.

Hoffman B. y Gallaher T. 2007. Importance indices in ethnobotany. Ethnobotany Research and Applications 5:201-218.

Kirchhoff P. 1943. Mesoamerica. Acta Americana 1:92-107. 
Lajones B.D.A. y Lema T.A. 1999. Propuesta y evaluación de un índice de valor de importancia etnobotánica por medio del análisis de correspondencia en las comunidades Arenales y San Salvador, Esmeraldas, Ecuador. Crónica Forestal y del Medio Ambiente 14:59-80.

León-Portilla M. 2005. Aztecas-Mexicas: Desarrollo de una Civilización Originaria. Algaba, Madrid.

Luna S.A. 1996. La flora representada en la iconografía pictórica. En: de la Fuente B. Coord. La Pintura Mural Prehispánica en México. I Teotihuacan, Tomo II, pp. 369-399, Instituto de Investigaciones Estéticas, Universidad Nacional Autónoma de México, México, D.F.

Manzanilla L.R. 2011. Metrópolis prehispánicas e impacto ambiental: el caso de Teotihuacan a través del tiempo. En: Caballero M. y Ortega G.B. Comps. Escenarios de Cambio Climático: Registros del Cuaternario en América Latina I, pp. 287-320, Instituto de Geofísica, Instituto de Geología, Dirección General de Publicaciones y Fomento Editorial, Universidad Nacional Autónoma de México, México, D.F.

Matos M.E. 1995. La Pirámide del Sol, Teotihuacan. Artes de México, Instituto Cultural Domeq, México, D.F.

McClung H.E.S. 1980. Interpretación de restos botánicos procedentes de sitios arqueológicos. Anales de Antropología 17:149-166.

McClung H.E.S. 2009. Los ecosistemas del valle de Teotihuacan a lo largo de su historia. En: Instituto Nacional de Antropología e Historia. Teotihuacan: Ciudad de los Dioses, pp. 37-45, Instituto Nacional de Antropología e Historia, México, D.F.

McClung H.E.S., Tapia-McClung R. y Gorenflo L.J. 2013. Biodiversity and landscape development in the Teotihuacan valley, basin of Mexico. Lessons from ecohistory for modern society. Journal of Cultural Symbiosis Research 8:185-192.

Millon R. 1970. Teotihuacan: completion of map of giant ancient city in valley of México. Science 170:1077-1082.

Monroy-Ortiz C. y Monroy R. 2004. Análisis preliminar de la dominancia cultural de las plantas útiles en el estado de Morelos. Boletín de la Sociedad Botánica de México 74:77-95.

Navarro P.L.C. y Avendaño R.S. 2002. Flora útil del municipio de Astacinga, Veracruz, México. Polibotánica 14:67-84.

Reyes-García V., Huanca T., Vadez V., Leonard W. y Wilkie D.
2006. Cultural, practical, and economic value of wild plants: A quantitative study in the Bolivian Amazon. Economic Botany 60:62-74.

Sahagún B. 1980. Historia General de las cosas de la Nueva España. Manuscrito 218-220 de la Colección Palatina de la Biblioteca Medicea Laurenziana, Florencia [reproducción facsimilar: Códice Florentino]. Archivo General de la Nación, México, D.F.

Sarukhán J., Koleff P., Carbias J., Soberón J., Dirzo R., Llorente-Bousquets J., Halffter G., González R., March I., Mohar A., Anta S. y de la Maza J. 2009. Capital Natural de México. Síntesis: Conocimiento Actual, Evaluación y Perspectivas de Sustentabilidad. Comisión Nacional para el Conocimiento y Uso de la Biodiversidad, México, D.F.

SAS Institute Inc. 2010. User's guide: statistics version 9.3. SAS Institute Inc., Cary.

Schultes R.E. y von Reis S. 1995. Ethnobotany Evolution of a Discipline. Dioscorides Press, Portland.

SEMARNAT. Secretaría de Medio Ambiente y Recursos Naturales. 2010. Norma Oficial Mexicana NOM-059- SEMARNAT2010. Protección ambiental-Especies nativas de México de flora y fauna silvestres-Categorías de riesgo y especificaciones para su inclusión, exclusión o cambio-Lista de especies en riesgo.

Suárez A., Williams-Linera G., Trejo C., Valdez-Hernández J.I., Cetina-Alcalá V.M. y Vibrans H. 2012. Local knowledge helps select species for forest restoration in a tropical dry forest of central Veracruz, Mexico. Agroforestry Systems 85:35-55.

Takhtajan A. 1997. Diversity and Classification of Flowering Plants. Columbia University Press, Nueva York.

Villaseñor J.L., Ortiz E. y Redonda-Martínez R. 2008. Catálogo de Autores de Plantas Vasculares de México. $2^{\mathrm{a}}$ ed. Instituto de Biología, Universidad Nacional Autónoma de México, Comisión Nacional para el Conocimiento y Uso de la Biodiversidad, México, D.F.

Zizumbo-Villareal D., Flores-Silva A. y Colunga-García M.P. 2012. The archaic diet in Mesoamerica: incentive for milpa development and species domestication. Economic Botany 66:328-343.

Recibido: 20 de agosto de 2013

Aceptado: 17 de noviembre de 2013 
Apéndice 1. Listado de posibles especies útiles teotihuacanas.

Usos: $\mathrm{Co}=$ comestible, $\mathrm{Cb}=$ combustible, $\mathrm{Ma}=$ materiales (ut, utensilios; ma, madera; fi, fibra; la, látex; mu, mucílago; re, resina), $\mathrm{Me}=$ medicinal, $\mathrm{Soc}=$ uso social (rit, ritual; al, alucinógeno). Fuentes: $\mathrm{CF}=$ Códice Florentino, Ec $=$ estudios etnobotánicos contemporáneos.

\begin{tabular}{|c|c|c|c|c|c|}
\hline Familia & $\begin{array}{l}\text { Taxa en reportes } \\
\text { arqueológicos }\end{array}$ & $\begin{array}{l}\text { Usos en } \\
\text { Teotihuacan } \\
\text { prehispánico }\end{array}$ & & & Posibles especies utilizadas por la cultura teotihuacana \\
\hline Agavaceae & Agave spp. & $\begin{array}{l}\text { Co, Cb, Ma (fi), } \\
\text { Soc (rit) }\end{array}$ & $\times$ & $\times$ & $\begin{array}{l}\text { Agave americana L. } \\
\text { Agave applanata Lem. ex Jacobi } \\
\text { Agave inaequidens K. Koch } \\
\text { Agave lechuguilla Torr. }\end{array}$ \\
\hline Agavaceae & Agave atrovirens & Soc (rit) & $\times$ & $\times$ & Agave atrovirens Karw. ex Salm-Dyck \\
\hline Agavaceae & Agave mapisaga & Co & & $\times$ & Agave mapisaga Trel. \\
\hline Agavaceae & Agave salmiana & Co & $\times$ & $\times$ & Agave salmiana Otto ex Salm-Dyck \\
\hline Agavaceae & indet. Liliaceae & $\mathrm{Ma}(\mathrm{fi})$ & $\times$ & $x$ & Yucca filifera Chabaud \\
\hline Amaranthaceae & Amaranthus spp. & Co, Soc (rit) & $x$ & $\begin{array}{l}\times \\
\times\end{array}$ & $\begin{array}{l}\text { Amaranthus hybridus L. } \\
\text { Amaranthus hypochondriacus L. }\end{array}$ \\
\hline Anacardiaceae & Spondias sp. & $\mathrm{Co}$ & $\begin{array}{l}\times \\
\times\end{array}$ & $\begin{array}{l}\times \\
\times\end{array}$ & $\begin{array}{l}\text { Spondias mombin L. } \\
\text { Spondias purpurea L. }\end{array}$ \\
\hline Asteraceae & Baccharis spp. & $\mathrm{Cb}$ & & $\begin{array}{l}\times \\
\times \\
\times\end{array}$ & $\begin{array}{l}\text { Baccharis conferta Kunth } \\
\text { Baccharis pteronioides DC. } \\
\text { Baccharis salicifolia (Ruiz \& Pav.) Pers. } \\
\text { Baccharis sordescens DC. }\end{array}$ \\
\hline Asteraceae & Senecio salignus & $\mathrm{Cb}$ & & $x$ & Barkleyanthus salicifolius (Kunth) H.Rob. \& Brettell \\
\hline Betulaceae & Alnus spp. & $\mathrm{Cb}$ & & $\times$ & $\begin{array}{l}\text { Alnus acuminata Kunth } \\
\text { Alnus acuminata Kunth subsp. glabrata } \\
\text { (Fernald) Furlow } \\
\text { Alnus jorullensis Kunth }\end{array}$ \\
\hline Buddlejaceae & $\begin{array}{l}\text { Buddleia cf. } \\
\text { parviflora }\end{array}$ & $\mathrm{Cb}$ & & $x$ & Buddleja parviflora Kunth \\
\hline Burseraceae & (copal) & Soc (rit) & & $\begin{array}{l}\times \\
\times \\
\times \\
\times\end{array}$ & $\begin{array}{l}\text { Bursera bipinnata (Moc. \& Sessé ex DC.) Engl. } \\
\text { Bursera copallifera (Sessé \& Moc. ex DC.) Bullock } \\
\text { Bursera cuneata (Schltdl.) Engl. } \\
\text { Bursera fagaroides (Kunth) Engl. }\end{array}$ \\
\hline Cactaceae & Echinocactus sp. & Soc (rit) & $x$ & $\times$ & Echinocactus horizonthalonius Lem. \\
\hline Cactaceae & Ferocactus sp. & Soc (rit) & $\times$ & $\times$ & Ferocactus latispinus (Haw.) Britton \& Rose \\
\hline Cactaceae & Heliocereus sp. & Soc (rit) & & & Heliocereus speciosus (Cav.) Britton \& Rose \\
\hline Cactaceae & $\begin{array}{l}\text { Mammillaria } \\
\text { magnimamma }\end{array}$ & Soc (rit) & & $\times$ & Mammillaria magnimamma Haw. \\
\hline Cactaceae & Mammillaria sp. & Soc (rit) & & $\times$ & $\begin{array}{l}\text { Mammillaria atrorubra Ehrenb. } \\
\text { Mammillaria purpurea Ehrenb. } \\
\text { Mammillaria rhodantha Link \& Otto subsp. Rhodantha } \\
\text { Mammillaria uncinata Zucc. ex Pfeiff. } \\
\text { Mammillaria wiesingeri Boed. subsp. wiesingeri }\end{array}$ \\
\hline Cactaceae & $\begin{array}{l}\text { Myrtillocactus } \\
\text { geometrizans }\end{array}$ & Co & & $\times$ & Myrtillocactus geometrizans (Mart. ex Pfeiff.) Console \\
\hline Cactaceae & Opuntia spp. & $\begin{array}{l}\text { Co, Ma (mu), } \\
\text { Soc (rit) }\end{array}$ & $\times$ & $\times$ & Opuntia spp. \\
\hline Cactaceae & Opuntia amyclaea & Co & $\times$ & & Opuntia amyclaea Ten. \\
\hline Cactaceae & Opuntia cochinera & $\mathrm{Co}$ & & & Opuntia cochinera Griffiths \\
\hline Cactaceae & Opuntia matudae & Co & & & Opuntia matudae Scheinvar \\
\hline Cactaceae & $\begin{array}{l}\text { Opuntia } \\
\text { oligacantha }\end{array}$ & $\mathrm{Co}$ & & $\times$ & Opuntia oligacantha Hort. ex Pfeiff. \\
\hline
\end{tabular}


Apéndice 1. Continuación

\begin{tabular}{|c|c|c|c|c|c|}
\hline \multirow[t]{2}{*}{ Familia } & \multirow{2}{*}{$\begin{array}{l}\text { Taxa en reportes } \\
\text { arqueológicos }\end{array}$} & \multirow{2}{*}{$\begin{array}{l}\text { Usos en } \\
\text { Teotihuacan } \\
\text { prehispánico }\end{array}$} & \multicolumn{2}{|c|}{ Fuente } & \multirow[t]{2}{*}{ Posibles especies utilizadas por la cultura teotihuacana } \\
\hline & & & $\mathrm{CF}$ & Ec & \\
\hline Cactaceae & $\begin{array}{l}\text { Opuntia robusta } \\
\text { var. guerrana }\end{array}$ & Co & & & $\begin{array}{l}\text { Opuntia robusta J.C. Wendl. var. guerrana } \\
\text { (Griffiths) Sánchez-Mej. }\end{array}$ \\
\hline Cactaceae & Opuntia sarca & Co & & $x$ & Opuntia sarca Griffiths ex Scheinvar \\
\hline Cactaceae & Opuntia spinulifera & Co & & & Opuntia spinulifera Salm-Dyck \\
\hline Cactaceae & $\begin{array}{l}\text { Opuntia } \\
\text { streptacantha }\end{array}$ & Co & $x$ & $x$ & Opuntia streptacantha Lem. \\
\hline Cactaceae & $\begin{array}{l}\text { Opuntia tomentosa } \\
\text { var. tomentosa }\end{array}$ & Co & & & Opuntia tomentosa Salm-Dyck var. tomentosa \\
\hline Chenopodiaceae & (huauzontle) & Co, Soc (rit) & & & $\begin{array}{l}\text { Chenopodium berlandieri Moq. subsp. nuttalliae } \\
\text { (Saff.) Wison \& Heiser }\end{array}$ \\
\hline Chenopodiaceae & Chenopodium sp. & Co, Me, Soc (rit) & & $\times$ & $\begin{array}{l}\text { Chenopodium berlandieri Moq. subsp. berlandieri } \\
\text { Chenopodium fremontii S.Watson } \\
\text { Chenopodium mexicanum Moq. }\end{array}$ \\
\hline & & & $x$ & $\times$ & Dysphania ambrosioides (L.) Mosyakin \& Clemants \\
\hline Convolvulaceae & Ipomoea sp. & Me & $\times$ & $\times$ & $\begin{array}{l}\text { Dysphania graveolens (Willd.) Mosyakin \& Clemants } \\
\text { Ipomoea capillacea (Kunth) G. Don }\end{array}$ \\
\hline & & & & $\times$ & Ipomoea murucoides Roem. \& Schult. \\
\hline & & & & $x$ & Ipomoea purpurea (L.) Roth \\
\hline & & & & $x$ & Ipomoea stans Cav. \\
\hline Convolvulaceae & Turbina corymbosa & Soc $(\mathrm{al})$ & $\times$ & $\times$ & Turbina corymbosa (L.) Raf. \\
\hline Cucurbitaceae & Cucurbita spp. & Co & $x$ & $\times$ & Cucurbita ficifolia Bouché \\
\hline & & & $\times$ & $x$ & Cucurbita pepo L. \\
\hline Cucurbitaceae & Lagenaria siceraria & Ma (ut) & $x$ & $x$ & Lagenaria siceraria (Molina) Standl. \\
\hline Cupressaceae & $\begin{array}{l}\text { Cupressus cf. } \\
\text { lindleyi }\end{array}$ & $\mathrm{Cb}$ & $\times$ & $\times$ & Cupressus lusitanica Mill. \\
\hline Cupressaceae & $\begin{array}{l}\text { Juniperus cf. } \\
\text { Deppeana }\end{array}$ & $\mathrm{Cb}$ & & $\times$ & Juniperus deppeana Steud. \\
\hline Cyperaceae & Scirpus sp. & Ma (ut) & & & $\begin{array}{l}\text { Schoenoplectus americanus (Pers.) Volkart } \\
\text { Schoenoplectus californicus (C.A.Mey.) Soják } \\
\text { Schoenoplectus tabernaemontani (C.C.Gmel.) Palla }\end{array}$ \\
\hline Cyperaceae & Cyperus sp. & Ma (ut) & & & $\begin{array}{l}\text { Cyperus hermaphroditus (Jacq.) Standl. } \\
\text { Cyperus seslerioides Kunth } \\
\text { Cyperus spectabilis Link }\end{array}$ \\
\hline Ericaceae & $\begin{array}{l}\text { Arbutus cf. } \\
\text { xalapensis }\end{array}$ & $\mathrm{Cb}$ & & $x$ & Arbutus xalapensis Kunth \\
\hline Fabaceae & Acacia farnesiana & $\mathrm{Cb}$ & $x$ & $x$ & Acacia farnesiana (L.) Willd. \\
\hline Fabaceae & $\begin{array}{l}\text { Eysenhardtia } \\
\text { polystachya }\end{array}$ & $\mathrm{Cb}$ & $\times$ & $\times$ & Eysenhardtia polystachya (Ortega) Sarg. \\
\hline Fabaceae & $\begin{array}{l}\text { Mimosa cf. } \\
\text { aculeaticarpa var. } \\
\text { biuncifera }\end{array}$ & $\mathrm{Cb}$ & & & $\begin{array}{l}\text { Mimosa aculeaticarpa Ortega var. biuncifera } \\
\text { (Benth.) Barneby }\end{array}$ \\
\hline Fabaceae & $\begin{array}{l}\text { Phaseolus } \\
\text { coccineus }\end{array}$ & Co & $x$ & $x$ & Phaseolus coccineus L. \\
\hline Fabaceae & Phaseolus vulgaris & Co & $x$ & $x$ & Phaseolus vulgaris L. \\
\hline Fabaceae & Prosopis laevigata & $\mathrm{Cb}$ & $\times$ & $\times$ & $\begin{array}{l}\text { Prosopis laevigata (Humb. \& Bonpl. ex Willd.) } \\
\text { M.C. Johnst. }\end{array}$ \\
\hline Fagaceae & Quercus spp. & $\mathrm{Cb}, \mathrm{Ma}(\mathrm{ma})$ & & $\begin{array}{l}\times \\
\times\end{array}$ & $\begin{array}{l}\text { Quercus crassipes Bonpl. } \\
\text { Quercus dysophylla Benth. }\end{array}$ \\
\hline
\end{tabular}


Apéndice 1. Continuación

\begin{tabular}{|c|c|c|c|c|c|}
\hline \multirow[t]{2}{*}{ Familia } & \multirow{2}{*}{$\begin{array}{l}\text { Taxa en reportes } \\
\text { arqueológicos }\end{array}$} & \multirow{2}{*}{$\begin{array}{l}\text { Usos en } \\
\text { Teotihuacan } \\
\text { prehispánico }\end{array}$} & \multicolumn{2}{|c|}{ Fuente } & \multirow[t]{2}{*}{ Posibles especies utilizadas por la cultura teotihuacana } \\
\hline & & & CF & Ec & \\
\hline & & & & $\times$ & Quercus greggii (A.DC.) Trel. \\
\hline & & & & $\times$ & Quercus laurina Bonpl. \\
\hline & & & & $\times$ & Quercus mexicana Bonpl. \\
\hline & & & & $\times$ & Quercus microphylla Née \\
\hline & & & & $\times$ & Quercus rugosa Née \\
\hline Hydrocotylaceae & $\begin{array}{l}\text { Hydrocotyle } \\
\text { ranunculoides }\end{array}$ & Soc (rit) & & $\times$ & Hydrocotyle ranunculoides L. f. \\
\hline Hydrocotylaceae & Hydrocotyle sp. & Me & & & Hydrocotyle verticillata Thunb. \\
\hline Lamiaceae & Salvia sp. & Co & $x$ & $x$ & Salvia hispanica L. \\
\hline Lauraceae & Persea sp. & Co & $\times$ & $\times$ & Persea americana Mill. \\
\hline Malvaceae & Gossypium sp. & $\mathrm{Ma}(\mathrm{fi})$ & $x$ & $x$ & Gossypium hirsutum L. \\
\hline Moraceae & Castilla elastica & Ma (la) & $\times$ & $\times$ & Castilla elastica Sessé \\
\hline Moraceae & Ficus sp. & $\mathrm{Ma}(\mathrm{fi})$ & & $\times$ & Ficus petiolaris Kunth \\
\hline Nolinaceae & indet. Liliaceae & Ma (fi) & & $x$ & Nolina parviflora (Kunth) Hemsl. \\
\hline Nymphaeaceae & $\begin{array}{l}\text { Nymphaea } \\
\text { mexicana }\end{array}$ & Soc (rit) & $\times$ & & Nymphaea mexicana Zucc. \\
\hline Onagraceae & $\begin{array}{l}\text { Fuchsia cf. } \\
\text { Arborescens }\end{array}$ & $\mathrm{Cb}$ & & $\times$ & Fuchsia arborescens Sims \\
\hline Papaveraceae & Papaver sp. & Me & & & $\begin{array}{l}\text { Argemone ochroleuca Sweet subsp. stenopetala } \\
\text { (Prain) G.B.Ownbey }\end{array}$ \\
\hline Pinaceae & Pinus leiophylla & $\mathrm{Cb}, \mathrm{Ma}(\mathrm{ma})$ & & $x$ & Pinus leiophylla Schiede ex Schltdl. \& Cham. \\
\hline Pinaceae & Pinus montezumae & Ma (re), Soc (rit) & & $x$ & Pinus montezumae Lamb. \\
\hline Pinaceae & Pinus spp. & $\begin{array}{l}\mathrm{Cb}, \mathrm{Ma}(\mathrm{ma}) \\
\text { Soc (rit) }\end{array}$ & $x$ & $\times$ & Pinus ayacahuite Ehrenb. ex Schltdl. \\
\hline Pinaceae & & & & $\times$ & Pinus cembroides Zucc. \\
\hline Pinaceae & & & & & Pinus devoniana Lindl. \\
\hline Pinaceae & & & & $x$ & Pinus hartwegii Lindl. \\
\hline Pinaceae & & & & & Pinus lawsonii Roezl ex Gordon \\
\hline Pinaceae & & & & $\times$ & Pinus patula Schiede ex Schltdl. \& Cham. \\
\hline Pinaceae & & & & $\times$ & Pinus pseudostrobus Lindl. \\
\hline Pinaceae & & & $\times$ & $x$ & Pinus teocote Schiede ex Schltdl. \& Cham. \\
\hline Poaceae & $\begin{array}{l}\text { Phragmites } \\
\text { australis }\end{array}$ & Ma (ut) & & & Phragmites australis (Cav.) Trin. ex Steud. \\
\hline Poaceae & Zea mays & Co, Soc (rit), Cb & $x$ & $\times$ & Zea mays L. \\
\hline Portulacaceae & Portulaca oleracea & Co & $x$ & $x$ & Portulaca oleracea L. \\
\hline Rosaceae & $\begin{array}{l}\text { Crataegus } \\
\text { mexicana }\end{array}$ & Co & $\times$ & $\times$ & Crataegus mexicana DC. \\
\hline Rosaceae & $\begin{array}{l}\text { Prunus serotina } \\
\text { Ehrh. subsp. capulí }\end{array}$ & $\begin{array}{l}\mathrm{Co}, \mathrm{Cb}, \mathrm{Me} \text {, } \\
\text { Soc (rit) }\end{array}$ & $\times$ & $\times$ & Prunus serotina Ehrh. subsp. capuli (Cav.) McVaugh \\
\hline Rutaceae & Casimiroa edulis & $\mathrm{Co}, \mathrm{Me}$ & $\times$ & $x$ & Casimiroa edulis La Llave \\
\hline Salicaceae & Salix spp. & $\mathrm{Cb}$ & & $\times$ & Salix bonplandiana Kunth \\
\hline Solanaceae & Capsicum sp. & Co, Soc (rit) & $x$ & $x$ & Capsicum annuum L. \\
\hline Solanaceae & Datura ceratocaula & Soc (al) & $\times$ & & Datura ceratocaula Ortega \\
\hline Solanaceae & Datura stramonium & Soc (al) & $\times$ & $\times$ & Datura stramonium L. \\
\hline Solanaceae & indet. (jitomate) & Co & $\times$ & $\times$ & Solanum lycopersicum L. \\
\hline Solanaceae & Physalis sp. & Co, Me, Soc (rit) & & $x$ & $\begin{array}{l}\text { Physalis chenopodifolia Lam. var. chenopodifolia } \\
\text { Physalis orizabae Dunal } \\
\text { Physalis patula Mill. }\end{array}$ \\
\hline
\end{tabular}


Mariana T. VÁzquez-Alonso et aL.

Apéndice 1. Continuación

\begin{tabular}{|c|c|c|c|c|c|}
\hline \multirow[t]{2}{*}{ Familia } & \multirow{2}{*}{$\begin{array}{l}\text { Taxa en reportes } \\
\text { arqueológicos }\end{array}$} & \multirow{2}{*}{$\begin{array}{l}\text { Usos en } \\
\text { Teotihuacan } \\
\text { prehispánico }\end{array}$} & \multicolumn{2}{|c|}{ Fuente } & \multirow[t]{2}{*}{ Posibles especies utilizadas por la cultura teotihuacana } \\
\hline & & & CF & Ec & \\
\hline & & & & $\times$ & Physalis philadelphica Lam. \\
\hline & & & $\times$ & $\times$ & Physalis pubescens L. \\
\hline \multirow[t]{2}{*}{ Solanaceae } & Solanum sp. & Co & & $\times$ & Solanum demissum Lindl. \\
\hline & & & & & Solanum stoloniferum Schltdl. \\
\hline Sterculiaceae & Theobroma сасао & Co, Soc (rit) & $\times$ & $x$ & Theobroma cacao L. \\
\hline \multirow[t]{2}{*}{ Taxodiaceae } & Taxodium & $\mathrm{Cb}$ & $\times$ & $\times$ & Taxodium mucronatum Ten. \\
\hline & mucronatum & & & & \\
\hline \multirow[t]{2}{*}{ Typhaceae } & Thypa latifolia & Ma (ut) & & & Typha latifolia L. \\
\hline & & & & & Typha domingensis Pers. \\
\hline Vitaceae & Cissus sicyoides & Me & & $\times$ & Cissus sicyoides L. \\
\hline
\end{tabular}

